\title{
Investigating the Mechanism of Lithium Transport at Solid Electrolyte Interphases
}

Ryan Jorn, Lauren Raguette, Shaniya Peart

Department of Chemistry, Villanova University, Villanova, Pennsylvania 19085, United States

\section{Supporting Information}

Determining the location of the film surface: For each simulation considered, the location of the interface between the electrolyte and the SEI film was determined by plotting the density of the SEI moieties and the EC center-of-mass particles. The intersection of these two densities was taken to correspond to the "interface" for reference purposes.

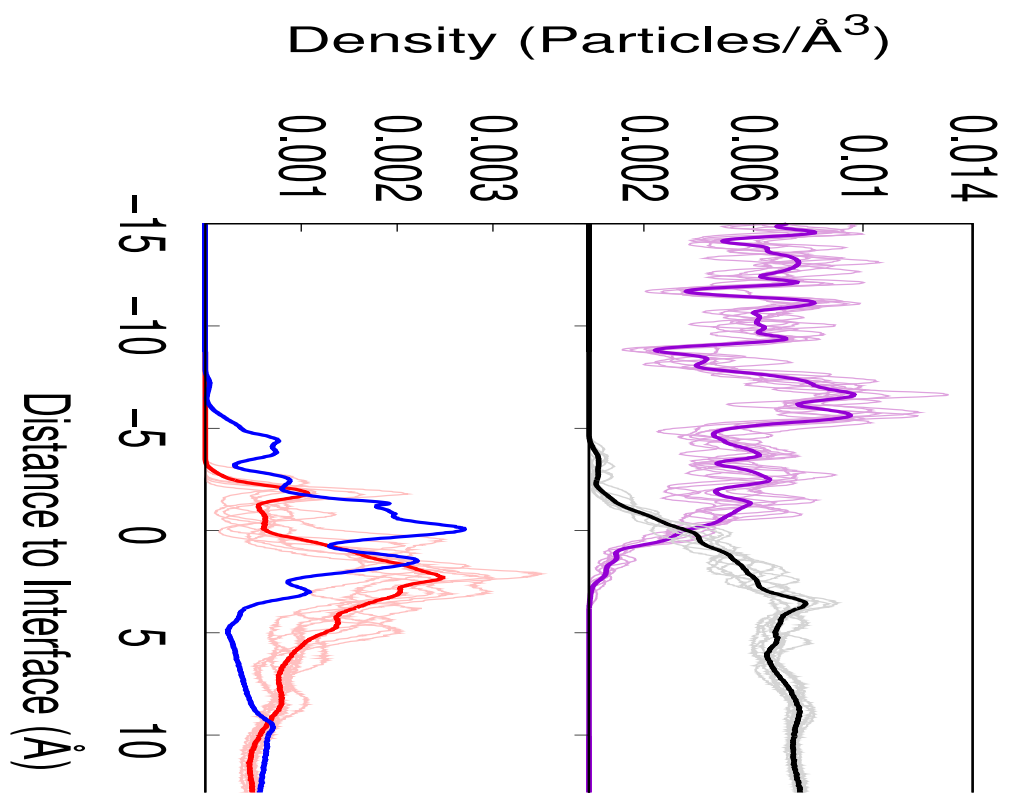

Figure S1. The center-of-mass density for $\mathrm{EDC}^{2-}$ (purple lines) and EC (black curves) molecules are shown in the upper panel for representative umbrella sampling simulations along Path 1 at the LEDC surface. The densities for the electrolyte $\mathrm{Li}^{+}$(blue curve) and center-of-mass for $\mathrm{PF}_{6}^{-}$(red curves) are shown for simulations along Path 1 in the lower panel. The bolded curves are the averages from all of the windows and the lighter curves correspond to individual window simulations. 


\section{Histograms for Potential of Mean Force Curves:}

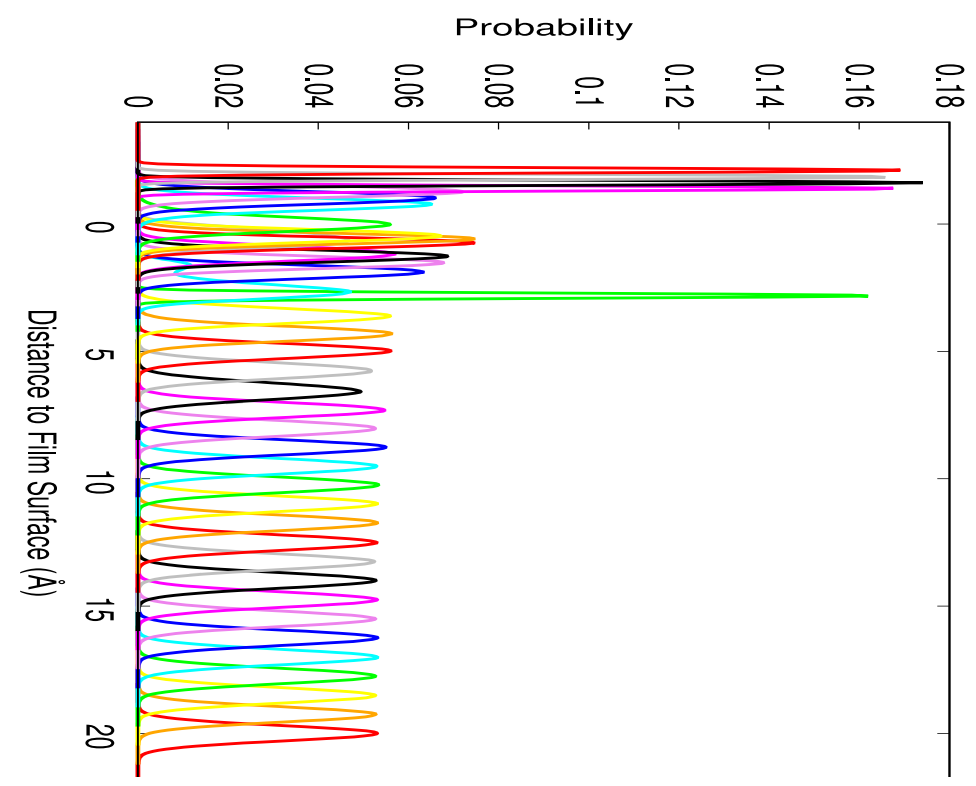

Figure S2. Probability distributions are shown for the lithium location in each of the windows of the umbrella sampling for Figure 3 along Path 1 at LEDC.

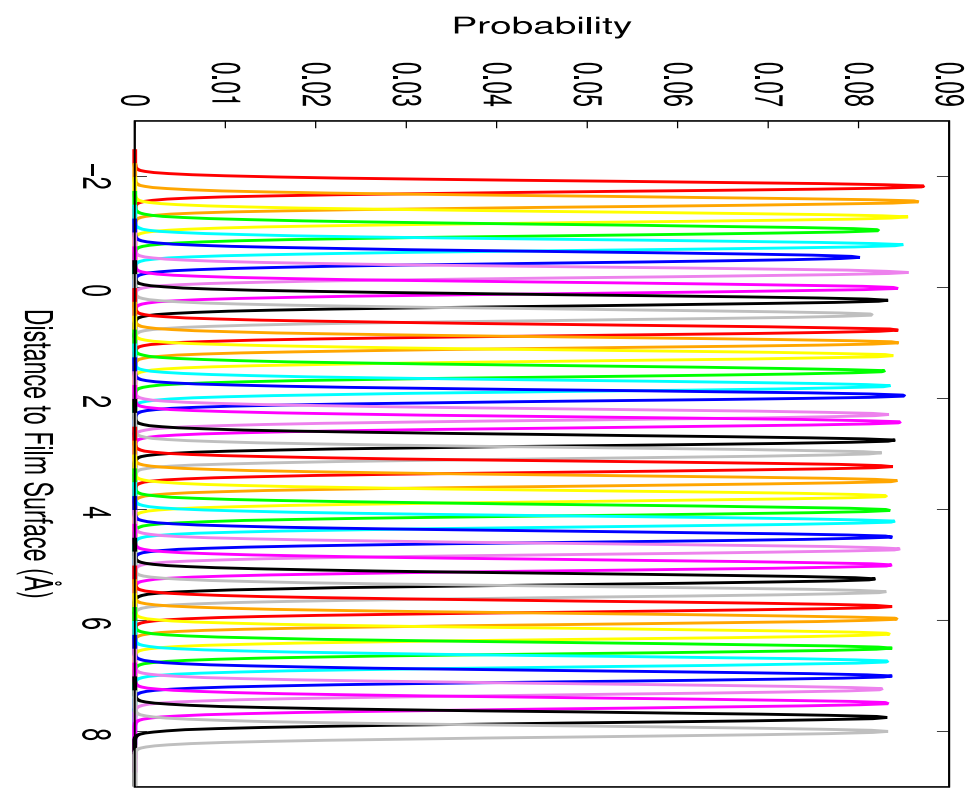

Figure S3. Probability distributions are shown for the lithium location in each of the windows of the umbrella sampling for Figure 5 of Path 2 at LEDC. 


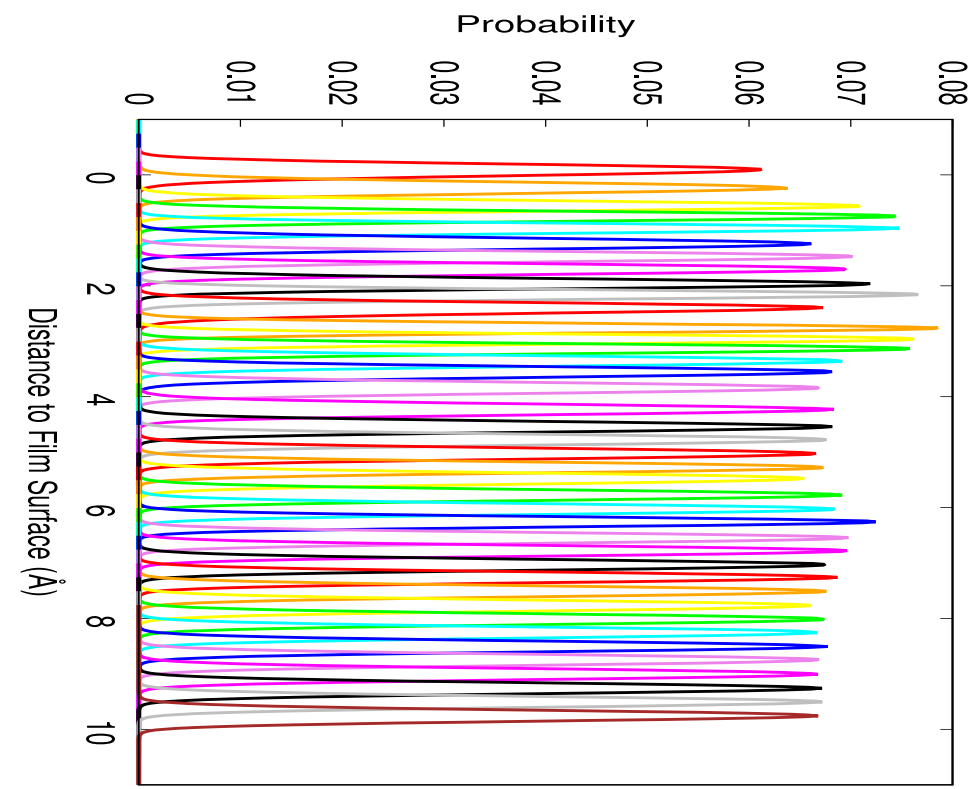

Figure S4. Probability distributions are shown for the lithium locations in each of the windows of the umbrella sampling of extraction from $\mathrm{Li}_{2} \mathrm{CO}_{3}$.

Changes in Electrolyte Li Ion Content Across Windows: In order to distinguish net charge transfer to the SEI from exchange of lithiums, the lithium ion density profile in the electrolyte region of select simulations was integrated. Agreement between lithium ion counts in the first and last umbrella sampling window shows that the biased lithium did not change the distributions in the electrolyte, i.e. the biased lithium exchanged positions with other lithiums in the simulation.

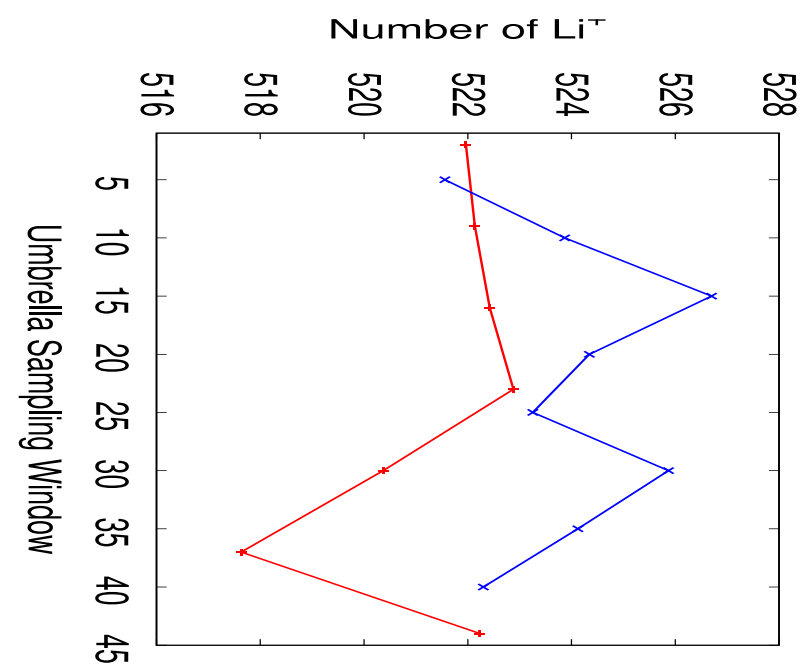

Figure S5. Integrated lithium ion densities for selected simulation windows from the umbrella sampling along Path 1 (red) and Path 2 (blue) at the LEDC interface. The curves correspond to windows from Figure 3 and 5 , respectively. 


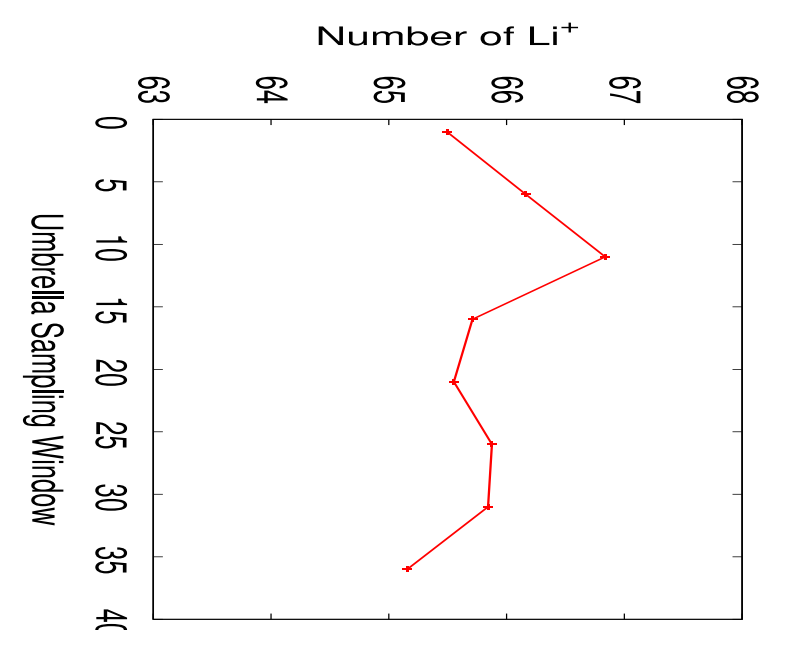

Figure S6. Integrated lithium ion densities for selected simulation windows from the umbrella sampling along Path 2 at the Li2CO3 interface. The number of lithium ions found differs from Figure S5 as a result of the frozen carbonate lithium ions being neglected in the integration.

\section{Additional Potential of Mean Force Curves for Lithium Ions along Path 1 @ LEDC:}

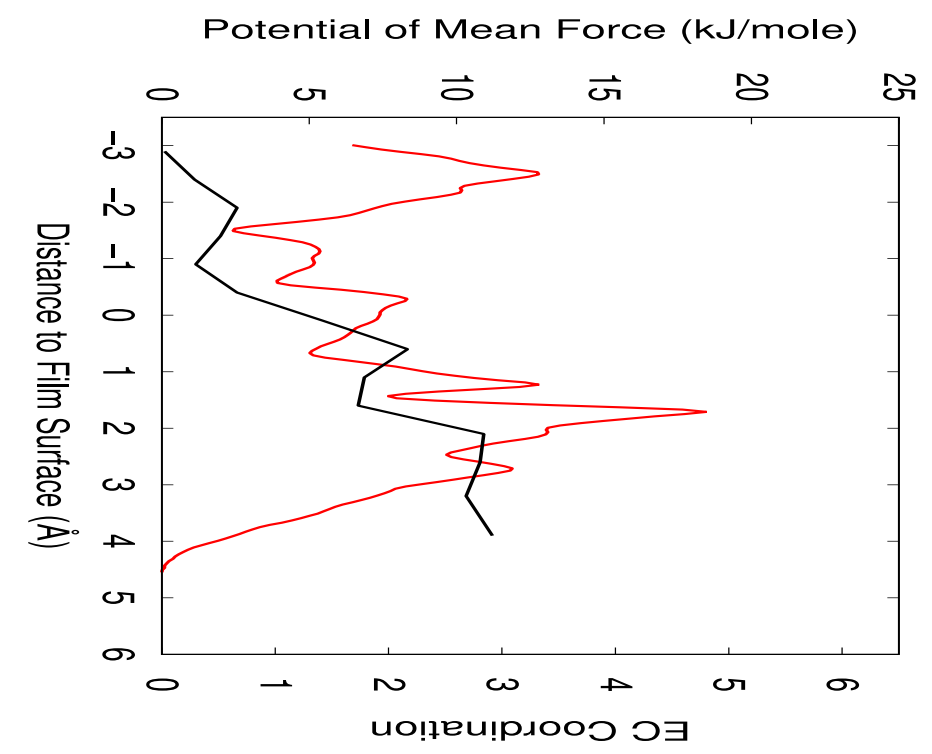

Figure S7. The PMF for lithium ion insertion (red) is shown along with the changes to EC coordination (black) along Path 1 for the LEDC surface using a different lithium ion from that shown in Figure 2. 


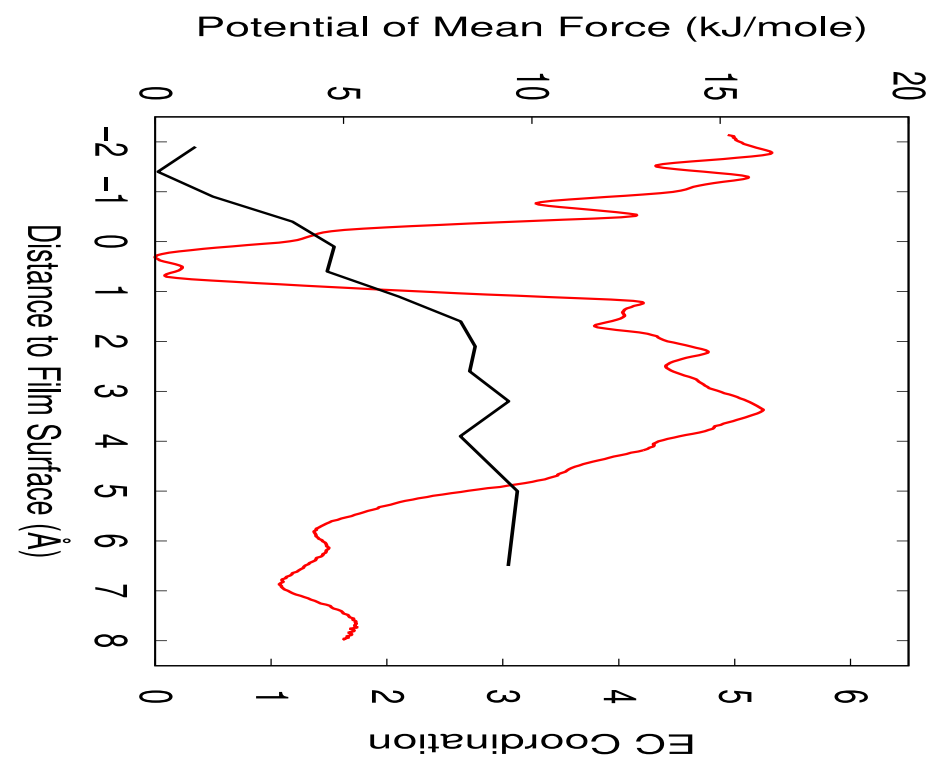

Figure S8. The PMF for lithium ion insertion (red) is shown along with the changes to EC coordination (black) along Path 1 for the LEDC surface using a different lithium ion from that shown in Figure 2 or Figure S5. 